\title{
Varying Effects of Corneal Thickness on Intraocular Pressure Measurements with Different Tonometers
}

\begin{abstract}
Aims To compare the intraocular pressure (IOP) measurements using the Goldmann applanation tonometer (GAT), noncontact tonometer (NCT), and ocular blood flow tonometer (OBFT), and to evaluate the effects of varying central corneal thickness (CCT) on the readings.

Methods Ultrasound pachymetry and tonometry were performed on $\mathbf{1 7 0}$ eyes. Using the corrected GAT values that took CCT into account as the standard, we calculated the NCT and OBFT measurement errors related to the CCT variable by a linear regression model. Group comparisons were performed with the $\chi^{2}$ test and one-way ANOVA test. The correlation between the various tonometer measurements was analysed by Pearson's correlation method.
\end{abstract}

Results Both the NCT $(r=0.872, P<0.001)$ and OBFT measurements $(r=0.861, P<0.001)$ were highly correlated with the GAT measurements. IOP measurements using the three tonometers were all correlated with CCT (all $P<0.001$ ), with the NCT measurements showing the greatest regression coefficient $(\beta=0.063, r=0.650)$ and the GAT measurements the least $(\beta=0.037, r=0.496)$. A linear regression model indicated that a $10 \mu \mathrm{m}$ change in CCT resulted in a NCT measurement deviation of $0.47-0.98 \mathrm{mmHg}$ and an OBFT measurement deviation of 0.29$0.81 \mathrm{mmHg}$.

Conclusion Pressure readings with the GAT, NCT, and OBFT are all affected by CCT, with the NCT being the one most affected and the GAT the least. Our findings suggest CCT an essential variable to consider in interpreting

Y-C Ko ${ }^{1,2}, \mathrm{CJ}-\mathrm{IL} \mathrm{Liu}^{1,3,4}$ and W-M Hsu',3

IOP readings, especially for the NCT measurements.

Eye (2005) 19, 327-332. doi:10.1038/sj.eye.6701458

Published online 16 July 2004

Keywords: corneal thickness; intraocular pressure measurement; tonometry

\section{Introduction}

Accurate estimation of intraocular pressure (IOP) is important because it is an essential factor in precise patient classification in diagnosis and efficacy assessment of glaucoma treatment. Several studies have shown that 30$60 \%$ of ocular hypertension $(\mathrm{OH})$ and normal tension glaucoma (NTG) patients are misclassified and possibly improperly treated due to a corneal thickness that is significantly deviated from the norm. ${ }^{1-4}$ Goldmann applanation tonometry, in which the tonometer is calibrated based on a central corneal thickness (CCT) of $500 \mu \mathrm{m}$, has been the gold standard for IOP measurement for decades. ${ }^{5}$ Studies comparing measurements with manometry and tonometry indicate that measuring CCT is essential to properly interpret the results obtained with a Goldmann applanation tonometer (GAT) ${ }^{6-8}$ To estimate the true intraocular hydrostatic pressure from the GAT readings, several investigators have proposed some formulae, which we employed for the purposes of this study, that take CCT into account. ${ }^{6,7,9-11}$ If applying these correction formulae to the Ocular Hypertension Treatment Study cohort, the proportion of subjects with a baseline IOP $\leq 21 \mathrm{mmHg}$ in the randomly
${ }^{1}$ Department of Ophthalmology Taipei Veterans General Hospital

Taipei, Taiwan

${ }^{2}$ Institute of Clinical Medicine

National Yang-Ming

University

Taipei, Taiwan

${ }^{3}$ National Yang-Ming University School of Medicine Taipei, Taiwan

${ }^{4}$ Department of Ophthalmology ChiMei Medical Center Tainan, Taiwan

Correspondence: CJ. Liu Department of Ophthalmology Taipei Veterans General Hospital

No. 201, Section 2 Shih-Pai Road, Shih-Pai Taipei 11217, Taiwan

E-mail: jlliu@

vghtpe.gov.tw

Tel: + 886228757133

Fax: + 886228761351

Received: 21 August 2003 Accepted: 15 January 2004 Published online: 16 July 2004

The authors have no financial interest related to the manuscript 
chosen eye at entry would be $52 \%$, which would be only $10 \%$ if uncorrected by these formulae. ${ }^{4}$

The noncontact tonometer (NCT) is now widely used as a screening tool for glaucoma because corneal anaesthesia and direct corneal contact are not required to obtain a reading. NCT readings are highly correlated with those of GAT in the normal IOP range. ${ }^{12-14}$

However, little is known about the impact of CCT on the NCT measurements. Recently, the ocular blood flow tonometer (OBFT) has been introduced as another option for measuring IOP and pulsatile ocular blood flow. It comes with a disposable head that offers protection against the risk of disease transmission. The manufacturers claim that IOP measurements with the OBFT are not affected by variations in CCT, a statement that needs to be verified.

In order to test the effects of variation in CCT on readings from each of the tonometers described above, we compared the IOP measurements obtained with the GAT, NCT, and OBFT in control subjects and in patients with glaucoma or ocular hypertension, and then evaluated the relationship between CCT and these measurements. Additionally, we quantified the NCT and OBFT measurement errors related to the variation in CCT by using the CCT-corrected GAT values as the standard.

\section{Materials and methods}

Patients were recruited consecutively from the outpatient clinic of one physician (CJL). All subjects were 18 years or older, and verbal and written consent was obtained from each. Institutional Review Board (IRB) approval was also obtained for the study. All subjects had a normal cornea on biomicroscopy, a spherical equivalent between -5 and +3 dioptres, a keratometric astigmatism $<2$ dioptres, and a clear optic medium allowing fundus examination. Contact lens wearers and subjects with connective tissue disorders, ocular inflammation, or a history of ocular surgery were excluded. Control subjects had untreated GAT values $<21 \mathrm{mmHg}$ and no ocular abnormalities other than mild refractive error or senile cataract. Patients with primary open-angle glaucoma (POAG), chronic primary angle closure glaucoma (CACG), NTG or OH were enrolled if they met all the inclusion and exclusion criteria except for the glaucomatous optic neuropathy or IOP status.

One masked, experienced ophthalmologist (YK) performed the following examinations for each subject. IOP was first measured with the same NCT (Topcon CT60, Topcon Corporation, Tokyo, Japan) to get three reliable readings. After $10 \mathrm{~min}$, five consecutive measurements of CCT with one ultrasound pachymeter (DGH 1000, DGH Technology, Inc., Frazer, PA, USA) were taken, and the mean of the middle three readings (in terms of numeric value) was used for analysis. After allowing the patient to rest for another $10 \mathrm{~min}$, IOP was measured with the same GAT and with one OBFT (Ocular Blood Flow Ltd, Wiltshire, UK, software version 16.2). The GAT and OBFT measurements were performed in random order and separated by $15 \mathrm{~min}$. Three measurements were obtained in each eye for each tonometer and the mean was used for analysis. All the instruments were calibrated according to the manufacturers' guidelines.

The corrected GAT value was calculated in three ways according to the formulae (Appendix) proposed by Ehlers et $a l_{,}{ }^{5}$ Whitacre et $a l^{6}{ }^{6}$ and Doughty et $a l^{9}{ }^{9}$ respectively. The deviations of the NCT and OBFT readings from the corrected GAT values were calculated and correlated to the CCT variables.

\section{Statistical analysis}

If both eyes of one patient were eligible, then one eye was chosen at random for analysis. Data were analysed with SPSS for windows, version 10.0. Group comparisons were performed with the $\chi^{2}$-test for categorical variables and one-way ANOVA test for continuous variables. The correlation between the IOP measurements obtained with different methods was analysed by Pearson's correlation method. The NCT and OBFT measurements were each separately compared with the GAT measurements by a paired $t$-test. The correlation between CCT and IOP was evaluated by a linear regression analysis. The level of significance was set at $P<0.05$. For multiple comparisons, the alpha levels were adjusted with the Bonferroni post hoc test.

\section{Results}

A total of 56 control subjects and 114 patients (POAG: 25, CACG: 40, NTG: 38, OH:11), all Asians, were enrolled. The demographic and clinical data for these subjects are listed in Table 1. Patients with $\mathrm{OH}$ were younger $(P<0.001)$ and had thicker corneas $(P<0.001)$ than the other groups, while NTG patients had thinner corneas than the control subjects $(P=0.001)$.

Both the NCT $(r=0.872, P<0.001)$ and OBFT $(r=0.861$, $P<0.001)$ readings were highly correlated with the GAT readings. The NCT readings were higher than the GAT readings in control subjects $(P=0.001)$ and in patients with $\mathrm{OH}(P=0.001)$ and POAG $(P=0.004)$ (Table 2). The OBFT tended to have lower readings than the GAT, but the difference was not significant after Bonferroni correction in each subgroup (Table 2).

Since a negative correlation existed between age and CCT $(P<0.001)$ as well as between age and IOP $(P<0.001)$, age was adjusted while performing linear 
Table 1 Demographic and clinical data

\begin{tabular}{|c|c|c|c|c|c|c|}
\hline & Control & $\mathrm{OH}$ & $P O A G$ & $C A C G$ & NTG & P-value \\
\hline Number of patients & 56 & 11 & 25 & 40 & 38 & \\
\hline Gender (male/female) & $31 / 25$ & $4 / 7$ & $14 / 11$ & $19 / 21$ & $28 / 10$ & 0.102 \\
\hline Age (years, mean $\pm S D)$ & $60.5 \pm 16.1$ & $46.2 \pm 15.8$ & $62.7 \pm 14.3$ & $68.0 \pm 9.2$ & $64.4 \pm 11.9$ & $<0.001$ \\
\hline Eye (OD/OS) & $37 / 19$ & $7 / 4$ & $17 / 8$ & $23 / \overline{17}$ & $21 / 17$ & 0.76 \\
\hline $\mathrm{CCT}^{\mathrm{a}}(\mu \mathrm{m}$, mean $\pm \mathrm{SD})$ & $567 \pm 43$ & $610 \pm 25^{\mathrm{b}}$ & $560 \pm 35$ & $557 \pm 35$ & $535 \pm 33^{\mathrm{b}}$ & $<0.001$ \\
\hline Range of CCT & $470-659$ & $572-663$ & $493-649$ & $479-623$ & $425-579$ & \\
\hline Number of glaucoma medication & 0 & $0.09 \pm 0.3$ & $0.9 \pm 0.96$ & $1.12 \pm 0.33$ & $0.42 \pm 0.5$ & \\
\hline
\end{tabular}

a Mean CCT of all the enrolled subjects: $559.4 \pm 40.8 \mu \mathrm{m}$.

bSignificant difference after Bonferroni correction: the $\mathrm{OH}$ group was younger $(P<0.001)$, and had thicker corneas $(P<0.001)$ than the other groups; the NTG group had thinner corneas than the control group $(P=0.001)$.

$\mathrm{OH}$ : ocular hypertension; POAG: primary open-angle glaucoma; CACG: chronic primary angle closure glaucoma; NTG: normal tension glaucoma; CCT: central corneal thickness.

Table 2 Measurements with the Goldmann applanation tonometer (GAT), noncontact tonometer (NCT), and ocular blood flow tonometer (OBFT)

\begin{tabular}{|c|c|c|c|c|c|}
\hline & $\begin{array}{l}\text { GAT }(m m H g) \\
(m e a n \pm S D)\end{array}$ & $\begin{array}{c}\text { NCT }(m m H g) \\
(m e a n \pm S D)\end{array}$ & $\begin{array}{c}\text { OBFT }(m m H g) \\
(m e a n \pm S D)\end{array}$ & $\begin{array}{c}\text { GAT vs NCT } \\
(P \text {-value })\end{array}$ & $\begin{array}{c}\text { GAT vs OBFT } \\
\text { (P-value })\end{array}$ \\
\hline All & $15.6 \pm 3.7$ & $16.6 \pm 4.9$ & $14.9 \pm 4.5$ & $<0.001$ & $<0.001$ \\
\hline Control & $14.9 \pm 2.8$ & $16.2 \pm 4.4$ & $14.1 \pm 4.3$ & $0.001^{\mathrm{a}}$ & 0.033 \\
\hline $\mathrm{OH}$ & $22.3 \pm 3.0$ & $24.8 \pm 3.8$ & $21.0 \pm 4.0$ & $0.001^{\mathrm{a}}$ & 0.086 \\
\hline POAG & $18.0 \pm 2.6$ & $19.4 \pm 3.6$ & $17.2 \pm 3.8$ & $0.004^{\mathrm{a}}$ & 0.112 \\
\hline CACG & $15.6 \pm 3.8$ & $16.4 \pm 4.9$ & $15.0 \pm 4.1$ & 0.032 & 0.130 \\
\hline NTG & $13.2 \pm 2.4$ & $13.2 \pm 2.8$ & $12.7 \pm 3.8$ & 0.840 & 0.145 \\
\hline
\end{tabular}

aSignificant difference after Bonferroni correction.

OH: ocular hypertension; POAG: primary open-angle glaucoma; CACG: chronic primary angle closure glaucoma; NTG: normal tension glaucoma.

regression of IOP on CCT. The IOP measurements with the three methods were all significantly correlated with CCT (all $P<0.001$ ) (Figure 1). The NCT measurements showed the greatest regression coefficient $(\beta=0.063$, $r=0.650$ ), while the GAT measurements showed the least regression coefficient $(\beta=0.037, r=0.496)$. The deviations of the NCT and OBFT readings from the corrected GAT values were highly correlated with CCT, no matter which correction formula was used (all $\mathrm{P}<$ 0.001) (Figures 2 and 3). Every $10 \mu \mathrm{m}$ change in CCT would yield a $0.98 \mathrm{mmHg}$ deviation in NCT measurements $(r=0.896)$ and a $0.81 \mathrm{mmHg}$ deviation in OBFT measurements $(r=0.837)$ according to the formula proposed by Ehlers et al. ${ }^{5}$ Based on the GAT values corrected with the formulae of Doughty et $a l^{6}$ and Whitacre et al, ${ }^{9}$ a $10 \mu \mathrm{m}$ CCT change would result in a $0.65 \mathrm{mmHg}(r=0.801)$ and a $0.47 \mathrm{mmHg}(r=0.716)$ error in NCT measurements respectively, and a $0.48 \mathrm{mmHg}$ $(r=0.665)$ and a $0.29 \mathrm{mmHg}(r=0.517)$ deviation in OBFT measurements, respectively.

\section{Discussion}

The realization of a wide range of CCT in normal eyes and the advent of excimer laser refractive surgery

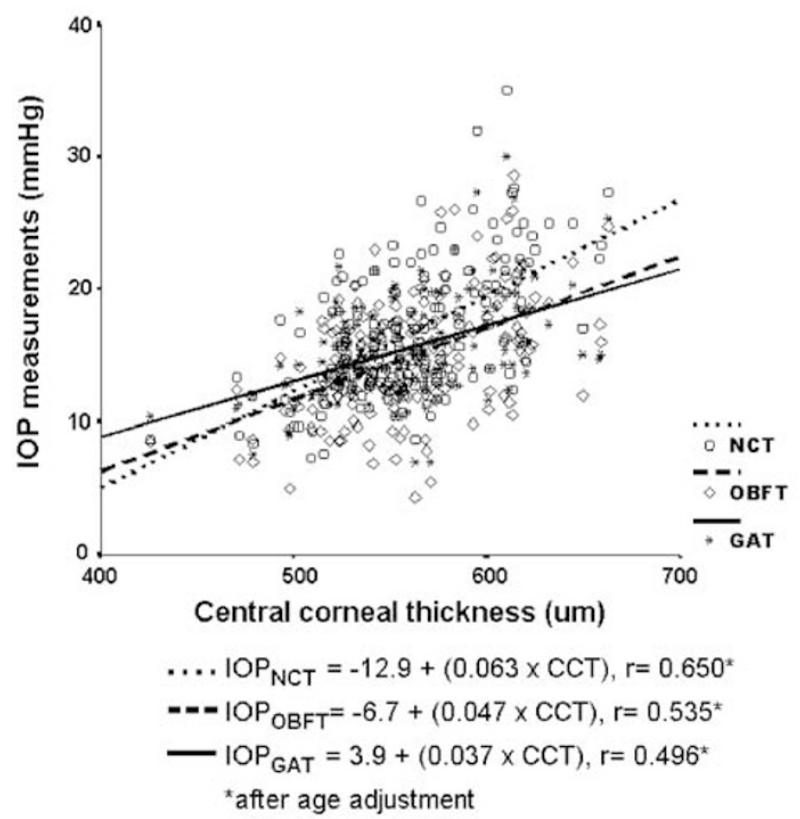

Figure 1 Correlation of CCT with the IOP measurements obtained with the NCT, OBFT and GAT.

prompted ophthalmologists to pay attention to the impact of CCT on IOP measurements. Although the 


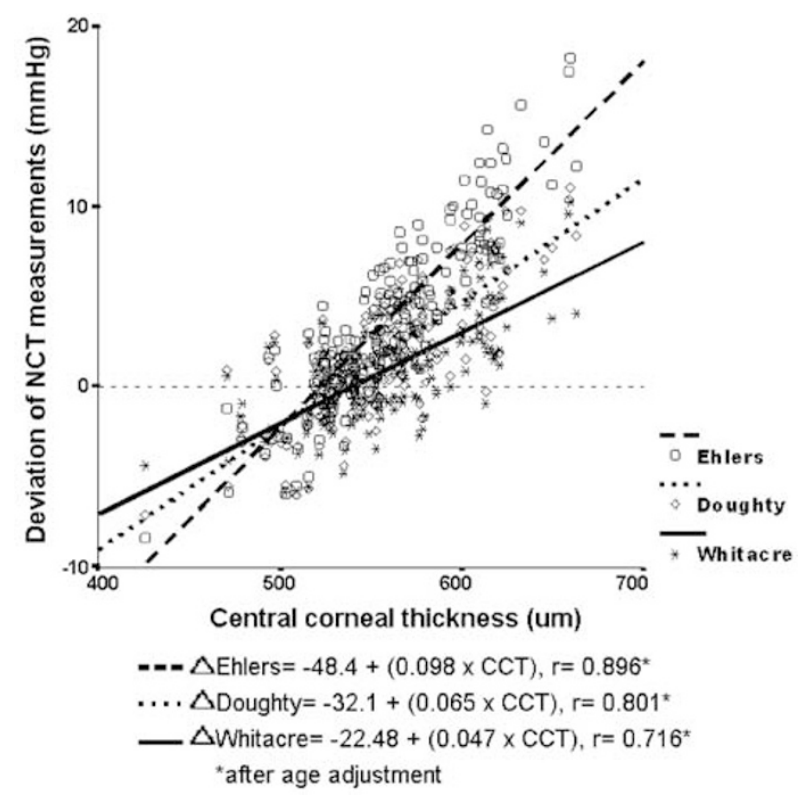

Figure 2 Correlation between CCT and the deviation of NCT measurements from the corrected GAT values. $\triangle$ : deviation of NCT measurements from the corrected GAT values that are calculated according to the formulae derived from the studies of Ehlers et al, Doughty et al, and Whitacre et al, respectively.

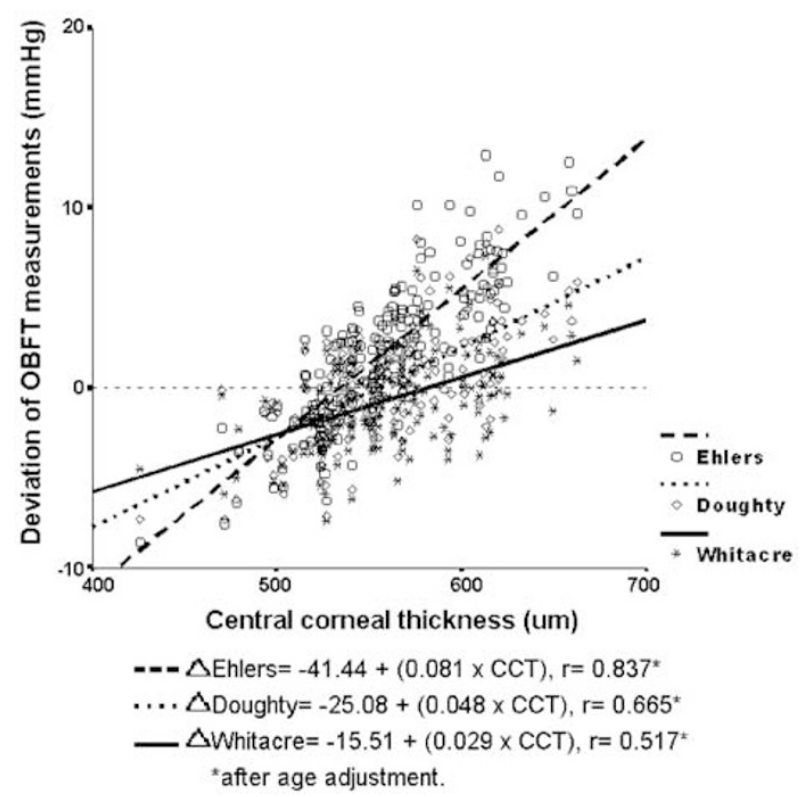

Figure 3 Correlation between CCT and the deviation of OBFT measurements from the corrected GAT values. $\triangle$ : deviation of OBFT measurements from the GAT values that are corrected according to the formulae derived from the studies of Ehlers et al, Doughty et al, and Whitacre et al, respectively.

CCT-induced error in GAT measurements has been quantitated by manometric studies, similar studies have never been performed with the NCT or OBFT in humans. Little is known about the degree to which the NCT or OBFT readings should be adjusted in eyes with a CCT significantly deviated from the population mean. We demonstrate that the measurements using the three methods are all influenced by CCT, with the NCT readings being the ones most affected and the GAT readings, the least. Using the corrected GAT value as a standard, we found that a $10 \mu \mathrm{m}$ change in CCT could yield a $0.47-0.98 \mathrm{mmHg}$ deviation in the NCT measurements and a $0.29-0.81 \mathrm{mmHg}$ deviation in the OBFT measurements.

The correlation between the NCT and GAT measurements has been widely explored, but articles dealing with the relationship between CCT and the NCT measurements are scanty. In a population study on subjects aged 50 years or older, Eysteinsson et $a l^{15}$ found that every $10 \mu \mathrm{m}$ increase in CCT is associated with a rise of $0.22-0.28 \mathrm{mmHg}$ in NCT (Nidek NT 2000) readings.

However, their study did not take into account individual variation in true intraocular hydrostatic pressure while estimating the influence of CCT on IOP readings. Therefore, those results might not truly reflect the amount of the CCT-induced error. Another study reported a significant intraindividual difference between the NCT (Topcon CT60) and GAT measurements in 60 glaucoma patients (120 eyes). ${ }^{16}$ The difference was positively correlated with CCT in eyes with a corneal thickness greater than $574.5 \mu \mathrm{m}$. This indicates that the CCT-induced overestimation is greater with the NCT than that with the GAT in eyes with a thick cornea. Our results support this conclusion. In addition, we found that the NCT readings could be overestimated by as great as $0.98 \mathrm{mmHg}$ with a $10 \mu \mathrm{m}$ increase in CCT. Conversely, the linear regression analyses demonstrate that the NCT readings could be underestimated in eyes with thin corneas.

The OBFT is a pneumotonometer that is designed by applying the elastic plate theory, and taking into account the radius and bending characteristics of the cornea. ${ }^{17}$ The manufacturer claims that the OBFT measurements are not affected by CCT, which is based on the results of two studies that use pneumotonometer to measure IOP before and after photorefractive keratectomy. ${ }^{18,19}$ However, our results show that the OBFT measurements are influenced by CCT. By analysing the relationship between CCT and the OBFT readings, several recent studies also showed that thicker corneas are associated with higher OBFT readings. ${ }^{20-22}$ Bhan et al ${ }^{20}$ found that the OBFT measurements were more affected by variations in CCT than the GAT and Tono-Pen measurements, with a mean increase in IOP with increasing CCT of $0.28 \mathrm{mmHg} / 10 \mu \mathrm{m}$. Morgan et al ${ }^{21}$ showed that a $10 \mu \mathrm{m}$ change in CCT was equivalent to a 
$0.3 \mathrm{mmHg}$ change in OBFT readings. Again, the abovementioned studies did not take individual pressure variations into account while evaluating the effect of CCT on OBFT measurements. In the present study, we found an error of $0.29-0.81 \mathrm{mmHg}$ in OBFT readings with a $10 \mu \mathrm{m}$ change in CCT.

This study recruited eyes with normal corneas, a spherical equivalent between -5.0 and 3.0 dioptres, and a keratometric astigmatism $<2$ dioptres. We did not consider the corneal curvature because the effect of it on applanation tonometer readings remains uncertain. Using 925 randomly sampled right eyes, Eysteinsson et $a l^{15}$ could not establish any relationship between the radius of corneal curvature and the NCT measurements. Paranhos et $a^{23}$ also found no significant influence of corneal curvature on IOP readings obtained with the GAT and NCT in 404 eyes with keratometric readings between 8.6 and $6.39 \mathrm{~mm}$. However, some suggested that corneal curvature should be taken as a correction factor in order to obtain true IOP.

Shimmyo et $a l^{24}$ derived a correction formula for adjusting GAT measurement by CCT and corneal curvature from statistical approximations. They found each millimetre of difference in corneal radius from an average of $7.85 \mathrm{~mm}$ caused $0.8 \mathrm{mmHg}$ of error in GAT measurements. Orssengo and $\mathrm{Pye}^{25}$ developed a theoretical mathematical model of the cornea, and proposed a correction factor between GAT measurement and true IOP according to corneal dimensions including central thickness and anterior radius of curvature. If applying Orssengo's and Shimmyo's formulae (Appendix) to calculate the corrected GAT values in our study cohort, ${ }^{24,25}$ a $10 \mu \mathrm{m}$ CCT change would result in a $0.59 \mathrm{mmHg}(r=0.728)$ and a $0.78 \mathrm{mmHg}(r=0.825)$ deviation in NCT measurements respectively, and a $0.45 \mathrm{mmHg}(r=0.642)$ and a $0.65 \mathrm{mmHg}(r=0.757)$ deviation in OBFT measurements respectively. Thus, the CCT-induced deviation in NCT or OBFT measurements from the corrected GAT values that are adjusted by both CCT and anterior corneal curvature is within the range derived from this study. This could probably be explained by the fact that the GAT measurement error induced by corneal curvature is much less than CCTinduced error, a notion supported by the studies of Shimmyo et al, and Orssengo and Pye.

In this study, we used the corrected GAT values as the standard to evaluate the impact of CCT on the NCT and OBFT measurements. Although this model may not be as accurate as a manometric study, it provides information which is more useful than that derived from simply correlating the relationship between CCT and IOP readings. Our findings may help clinicians interpret pressure readings obtained with the NCT and OBFT in each individual.
In conclusion, measurements with the GAT, NCT, and OBFT are all affected by variations in CCT, with the NCT readings being the ones most affected and the GAT readings, the least. In this way, our results concur with the previous studies and indicate the importance of adjusting IOP readings according to individual corneal thickness to avoid pressure overestimation or underestimation, both of which could lead to misclassification of a patient in diagnosis and affect the efficacy assessment of prescribed treatment, which is relevant and important in the clinical evaluation of glaucoma.

\section{References}

1 Argus WA. Ocular hypertension and central corneal thickness. Ophthalmology 1995; 102: 1810-1812.

2 Herndon LW, Choudhri SA, Cox T, Damji KF, Shields MB, Allingham RR. Central corneal thickness in normal, glaucomatous and ocular hypertensive eyes. Arch Ophthalmol 1997; 115: 1137-1141.

3 Copt RP, Thomas R, Mermoud A. Corneal thickness in ocular hypertension, primary open-angle glaucoma, and normal tension glaucoma. Arch Ophthalmol 1999; 117: 14-16.

4 Brandt JD, Beiser JA, Kass MA, Gordon MO, the Ocular Hypertension Treatment Study (OHTS) group. Central corneal thickness in the ocular hypertension treatment study (OHTS). Ophthalmology 2001; 108: 1779-1788.

5 Goldmann H, Schmidt T. Über applanationstonometrie. Ophthalmologica 1957; 134: 221-242.

6 Ehlers N, Bramsen T, Sperling S. Applanation tonometry and central corneal thickness. Acta Ophthalmol (Copenh) 1975; 53: 34-43.

7 Whitacre MM, Stein RA, Hassanein K. The effect of corneal thickness on applanation tonometry. Am J Ophthalmol 1993; 115: 592-596.

8 Johnson M, Kass MA, Moses RA, Grodzki WJ. Increased corneal thickness simulating elevated intraocular pressure. Arch Ophthalmol 1978; 96: 664-665.

9 Wolfs RC, Klaver CC, Vingerling JR, Grobbee DE, Hofman A, de Jong PTVM. Distribution of central corneal thickness and its association with intraocular pressure: the Rotterdam study. Am J Ophthalmol 1997; 123: 767-772.

10 Doughty MJ, Zaman ML. Human corneal thickness and its impact on intraocular pressure measures: a review and meta-analysis approach. Surv Ophthalmol 2000; 44: 367-408.

11 Bron AM, Creuzot-Garcher C, Goudeau-Boutillon S, d'Athis P. Falsely elevated intraocular pressure due to increased central corneal thickness. Graefe's Arch Clin Exp Ophthalmol 1999; 237: 220-224.

12 Forbes M, Pico G, Grolman B. A noncontact applanation tonometer. Arch Ophthalmol 1974; 91: 134-140.

13 Sørensen PN. The noncontact tonometer. Clinical evaluation on normal and diseased eyes. Acta Ophthalmol 1975; 53: 513-521.

14 Popovich KS, Shields MB. A comparison of intraocular pressure measurements with the XPERT noncontact tonometer and Goldmann applanation tonometry. J Glaucoma 1997; 6: 44-46.

15 Eysteinsson T, Jonasson F, Sasaki H, Arnarsson A, Sverrisson T, Sasaki K et al. Central corneal thickness, radius 
of the corneal curvature and intraocular pressure in normal subjects using non-contact techniques: Reykjavik eye study. Acta Ophthalmol Scand 2002; 80: 11-15.

16 Recep ÖF, Hasıripi HH, Çağıl N, Sarıkatipoğlu H. Relation between corneal thickness and intraocular pressure measurement by noncontact and applanation tonometry. J Cataract Refract Surg 2001; 27: 1781-1791.

17 Silver DM, Farrell RA. Validity of pulsatile ocular blood flow measurements. Surv Ophthalmol 1994; 38 (Suppl): S72-S80.

18 Abbasoglu OE, Bowman RW, Cavanagh HD, McCulley JP. Reliability of intraocular pressure measurements after myopic excimer photorefractive keratectomy. Ophthalmology 1998; 105: 2193-2196.

19 Tuunanen TH, Hämäläinen P, Mali M, Oksala O, Tervo T. Effect of photorefractive keratectomy on the accuracy of pneumatonometer readings in rabbits. Invest Ophthalmol Vis Sci 1996; 37: 1810-1814.

20 Bhan A, Browning AC, Shah S, Hamilton R, Dave D, Dua HS. Effect of corneal thickness on intraocular pressure measurements with the pneumotonometer, Goldmann applanation tonometer and Tono-pen. Invest Ophthalmol Vis Sci 2002; 43: 1389-1392.

21 Morgan AJ, Harper J, Hosking SL, Gilmartin B. The effect of corneal thickness and corneal curvature on pneumotonometer measurements. Curr Eye Res 2002; 25: 107-112.
22 Singh RP, Goldberg I, Graham SL, Sharma A, Mohsin M. Central corneal thickness, tonometry and ocular dimensions in glaucoma and ocular hypertension. J Glaucoma 2001; 10: 206-210.

23 Paranhos A, Paranhos FR, Prata JA, Omi CA, Mello PAA, Shields MB. Influence of keratometric readings on comparative intraocular pressure measurements with Goldmann, Tono-pen, and noncontact tonometers. J Glaucoma 2000; 9: 219-233.

24 Shimmyo M, Ross AJ, Moy A, Mostafavi R. Intraocular pressure, Goldmann applanation tension, corneal thickness, and corneal curvature in Caucasians, Asians, Hispanics and African Americans. Am J Ophthalmol 2003; 136: 603-613.

25 Orssengo GJ, Pye DC. Determination of the true intraocular pressure and modulus of elasticity of the human cornea in vivo. Bull Math Biol 1999; 61: 551-572.

\section{Appendix}

The corrected GAT value, representing the true intraocular hydrostatic pressure, was calculated according to the following formulae (see Table A1) derived from the studies of Ehlers et $a l_{,}^{5}$ Whitacre et $a l_{,}{ }^{6}$ Doughty et al, ${ }^{9}$ Shimmyo et $a l^{24}$ and Orssengo et al. ${ }^{25}$

Table A1 Corrected GAT Value

\begin{tabular}{|c|c|}
\hline Authors & Correction formulae \\
\hline Ehlers et al & Corrected GAT $=$ GAT $-(C C T-520) \times(5 / 70)$ \\
\hline Whitacre et al & Corrected GAT $=$ GAT $-(C C T-560) \times(2 / 100)$ \\
\hline \multirow[t]{4}{*}{ Doughty et al } & Normal eyes: \\
\hline & Corrected GAT $=$ GAT $-(\mathrm{CCT}-535) \times(1.1 / 50)$ \\
\hline & Glaucomatous eyes: \\
\hline & Corrected GAT $=\mathrm{GAT}-(\mathrm{CCT}-535) \times(2.5 / 50)$ \\
\hline \multirow{3}{*}{$\begin{array}{l}\text { Shimmyo et al } \\
\text { Orssengo et al }\end{array}$} & Corrected GAT $=\mathrm{GAT}+(550-\mathrm{CCT}) / 18 \mathrm{e}^{-0.005 \mathrm{GAT}}+0.8((675 / \mathrm{K} 1+\mathrm{K} 2)-7.848837)$ \\
\hline & Corrected GAT $=\frac{\text { GAT }}{\left(B_{\mathrm{c}}-C_{\mathrm{c}}+C\right) / \mathrm{B}}$ \\
\hline & $\begin{array}{l}B_{\mathrm{c}}=\left(0.6 \pi R_{\mathrm{c}}\left(R_{\mathrm{c}}-T_{\mathrm{c}} / 2\right) \sqrt{1-v^{2}}\right) / T_{\mathrm{c}}^{2}, C_{\mathrm{c}}=\left(\pi R_{\mathrm{c}}\left(R_{\mathrm{c}}-T_{\mathrm{c}} / 2\right)(1-v)\right) / A T_{\mathrm{c}} \\
B=\left(0.6 \pi R(R-T / 2) \sqrt{1-v^{2}}\right) / T^{2}, C=(\pi R(R-T / 2)(1-v)) / A T\end{array}$ \\
\hline
\end{tabular}

GAT: Goldmann applanation tonometer measurements $(\mathrm{mmHg})$; CCT: central corneal thickness measurements $(\mu \mathrm{m})$; $\mathrm{K} 1, \mathrm{~K} 2$ : keratometric readings (dioptre); $R_{c}$ : calibration value of radius of curvature of anterior cornea $(7.80 \mathrm{~mm}) ; T_{\mathrm{c}}$ : calibration value of central corneal thickness $(0.52 \mathrm{~mm}) ; v$ : Poisson's ratio of cornea ( 0.49 , dimensionless); $A$ : applanated area by Goldmann applanation tonometer $\left(7.35 \mathrm{~mm}^{2}\right)$; $R$ : radius of curvature of anterior cornea (mm); $T$ : central corneal thickness (mm). 